\title{
Spitzenforschung und -versorgung
}

Comprehensive, also "umfassend", soll Forschung und Patientenversorgung in den Exzellenz-Krebszentren sein. Um das zu gewährleisten, gibt die Deutsche Krebshilfe e. V. für jedes der nunmehr 13 Comprehensive Cancer Center auf einen Zeitraum von vier Jahren 3 Millionen Euro aus. Diesen Sommer haben sieben Zentren von der entsprechenden internationalen Gutachterkommission das Plazet erhalten (s. u.). Für sechs weitere stehe bald eine erneute Bewerbung als Spitzenzentrum an, teilt die Krebshilfe mit. Sie hob das Schwerpunktprogramm „Onkologische Spitzenzentren“ vor genau zehn Jahren aus der Taufe.

Martin Roos

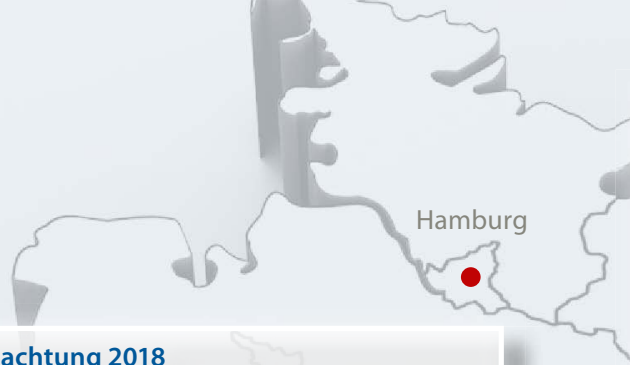

seit 2016 gefördert/weitergefördert

Berlin: Charité Comprehensive Cancer Center

Essen: Westdeutsches Tumorzentrum

Frankfurt/M: Universitäres Zentrum für Tumorerkrankungen

Freiburg: Comprehensive Cancer Center

Hamburg: Hubertus-Wald-Tumorzentrum

Mainz: Universitäres Centrum für Tumorerkrankungen

erneute Begutachtung 2018

Tübingen-Stuttgart: Südwestdeutsches Tumorzentrum

Dresden: Universitäts KrebsCentrum

Heidelberg: Nationales Centrum für Tumorerkrankungen

Köln/Bonn: Centrum für Integrierte Onkologie

München: Comprehensive Cancer Center

Ulm: Comprehensive Cancer Center

Würzburg: Comprehensive Cancer Center Mainfranken

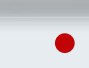

Essen
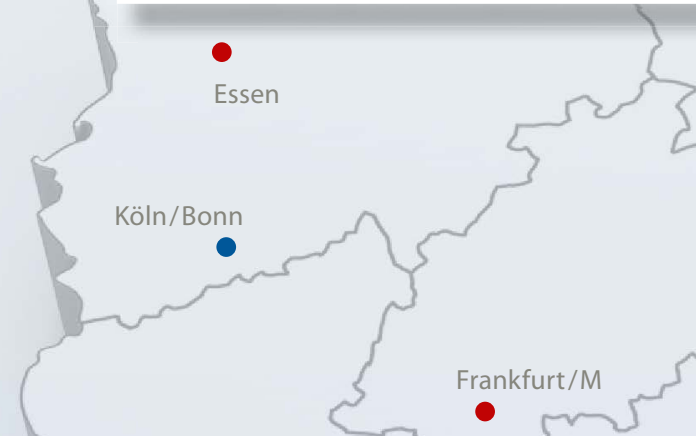

(n)

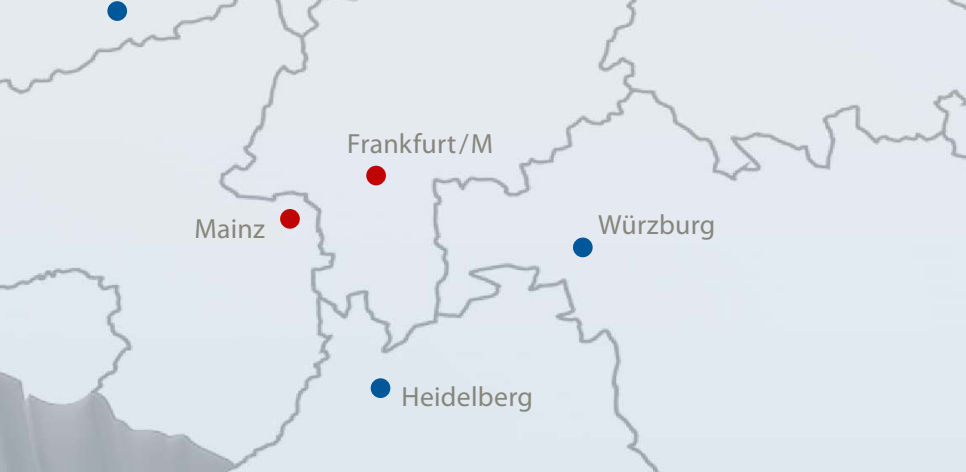

